\title{
Fomentar la resiliencia en familias con enfermedades crónicas pediátricas
}

\section{Building resilience in families with pediatric chronic diseases}

\section{Resumen}

En este artículo se describen los diferentes enfoques que se pueden aplicar al estudio del concepto de resiliencia familiar en el contexto de las enfermedades crónicas pediátricas y sus implicaciones en la práctica clínica y educativa. Asimismo, se describen los principios generales que deben regir esta práctica y las actuaciones concretas de los profesionales de los sistemas sanitarios, educativos y sociales. La resiliencia familiar es imprescindible para mantener una buena calidad de vida del niño enfermo y de la familia en su conjunto.

\section{Palabras clave}

Familia, resiliencia, enfermedad crónica pediátrica, terapia familiar, orientación familiar, intervención psicoeducativa.

\section{Abstract}

This article describes the different approaches that can be applied to the study of the concept of family resilience in the context of pediatric disease and their implications for clinical practice and education. It also describes the general principles that should govern this practice and the specific activities of the professional of health systems, educational and social. The resilience is essential to maintain a good quality of life of the sick and the family as a whole.

\section{Keywords}

Family, resilience, pediatric chronic illness, family therapy, family counseling, psychoeducational intervention.

\section{Claudia Grau Rubio \\ <Claudia.grau@uv.es>}

Departamento de Didáctica y

Organización Escolar, Universidad de Valencia

Instituto de Investigación Polibienestar, Universidad de Valencia

\footnotetext{
Para citar:

Grau Rubio, C. (20I3): "Fomentar la resiliencia en familias con enfermedades crónicas pediátricas", Revista Española de Discapacidad, I (I), I95-2I2.

doi: <http://dx.doi.org/IO.5569/23405IO4.OI.OI.IO>
}

Fecha de recepción: 27-IO-20I2 Fecha de aceptación: 22-4-20I3 


\section{Introducción}

En las últimas décadas se ha desarrollado el concepto de resiliencia familiar, con importantes repercusiones no sólo en el campo teórico, sino también en el de la práctica. En el ámbito de las enfermedades crónicas pediátricas, la resiliencia familiar puede entenderse como los procesos de adaptación y ajuste de la familia a las exigencias de la enfermedad (Patterson, 2002; McCubbin, 2002), que son diferentes en función de: si la enfermedad es aguda o gradual; si su curso es progresivo, constante o con recaídas; si supone un acortamiento de la vida o la muerte; si deriva en discapacidad o no; y si está en la fase de crisis, crónica o terminal (Rolland y Walsh, 2006). A su vez, estos procesos dependen de factores culturales, del género, el estatus socioeconómico, el contexto social y la historia familiar a lo largo de las generaciones, y del propio ciclo vital de la familia y de cada uno de sus miembros (Falicov, I995, 2007; McGoldrick, 2005; Masten, 2009); y hacen referencia al sistema de creencias familiar, a los patrones organizativos por los que se rige la familia, y al tipo de comunicación y de resolución de los problemas (Walsh, 2003).

En una enfermedad crónica intervienen un conjunto de profesionales provenientes de diferentes ámbitos: sanidad, educación y trabajo social. Los padres y el niño enfermo interactúan con ellos y, desde su ámbito de actuación, los profesionales pueden fomentar la resiliencia familiar a través de la colaboración con la familia, del desarrollo de las competencias necesarias para afrontar los problemas, de la confianza y del apoyo mutuo. Desde este enfoque positivo y pragmático, se fortalece el clima familiar, las posibilidades de trabajar juntos, la superación de obstáculos aparentemente insuperables y la consideración de que el éxito es debido, en gran parte, a sus esfuerzos, recursos y capacidades (Walsh, I996: 276; Villalba, 2003: 296).

\section{Concepto}

La resiliencia es un concepto proveniente de la física: es la capacidad que tienen los materiales de recobrar su forma original después de haber sido sometidos a una presión que los deforme. Este concepto fue aplicado a la psicología para describir la capacidad que tienen determinados sujetos y grupos de sobreponerse a las adversidades, de autorrregenerarse de determinadas heridas o traumas, y de sobreponerse a acontecimientos desestabilizadores; y el conjunto de procesos sociales y personales que permiten conseguir el bienestar físico y psicológico, a pesar de las adversidades.

A partir de los años setenta, las investigaciones se dirigen a aquellos niños que, en situaciones dramáticas (pobreza, violencia, maltrato, enfermedades crónicas y catástrofes), son capaces de llevar una vida productiva. Estas investigaciones se centraron, en una primera etapa, en describir las cualidades personales de estos individuos, tales como autonomía y autoestima (Walsh, 2003: I, 2; Black y Lobo, 2008: 35; Villalba, 2003: 287); en una segunda, en descubrir cómo se adquieren las cualidades resilientes y cuáles son los procesos asociados a una adaptación positiva (Villalba, 2003: 287); y en una tercera etapa, en qué es y dónde reside la fuente de energía o motivación para ser resilientes (Villalba, 2003: 29I).

Walsh (I996) elaboró el concepto de resiliencia familiar, entendiéndola como los procesos interactivos que fortalecen al individuo y a la familia en el transcurso del tiempo. Estos procesos interactivos son: reconocer los problemas y las limitaciones; hablar abierta y claramente acerca de ellos; analizar los recursos personales y familiares existentes; organizar y reorganizar las estrategias tantas veces como sea necesario, revisando y evaluando los logros y las pérdidas, con el objeto de reducir el estrés y la vulnerabilidad; promover la recuperación y el crecimiento después de la crisis; y fortalecer a las familias para superar la adversidad prolongada (Walsh, I996: 26I; 2002: I30, 2003: 2). 
Para esto es necesario que, en las relaciones entre los miembros de la familia, se produzcan: apoyos emocionales (relaciones de confirmación y confianza en la competencia de los protagonistas), conversaciones en las que se compartan objetivos (por ejemplo, acuerdos sobre premios y castigos), y se construyan significados acerca de la vida y de los acontecimientos adversos con coherencia narrativa y con un sentido dignificador para sus protagonistas (Walsh, 2003: 2). Así, los elementos básicos de la resiliencia familiar son: cohesión que no descarte la flexibilidad; comunicación franca entre los miembros de la familia; reafirmación de un sistema de creencias comunes; y resolución de problemas a partir de las anteriores premisas.

\section{Enfoques}

En el estudio de la resiliencia familiar existen varios enfoques, con importantes repercusiones en la práctica (Villalba, 2003: 293; Gómez y Kotliareno, 2010: ІІ3):

I. Resiliencia y respuesta familiar de ajuste y adaptación (Patterson, 2002; McCubbin, 2002).

2. Resiliencia y procesos clave (Walsh, 2003).

3. Sistema familiar y enfermedad (Rolland, I994).

4. Ecológico y de desarrollo (Falicov, I995; McGoldrick, 2005; Masten, 2009).

\subsection{Resiliencia y respuesta familiar de ajuste y adaptación}

El enfoque de resiliencia y respuesta familiar de ajuste y adaptación (Resilience Model of Family Stress, Adjustment and Adaptation) fue propuesto por McCubbin y McCubbin (I993), y Patterson (2002). Las familias con experiencias de estrés y sufrimiento por una enfermedad crónica pediátrica pueden dar sentido y propósito a su experiencia a través de lo positivo de sus fortalezas y relaciones con el contexto. Se adaptan combinando recursos, desarrollando estrategias de afrontamiento, valorando y resolviendo problemas, $\mathrm{y}$ haciendo ajustes a las nuevas demandas y estresores (McCubbin et al., 2002: I04; Patterson, 2002: 350 ; Walsh, 2003: 3).

Después de la crisis provocada por la enfermedad, la familia pasa por dos fases: la de ajuste y la de adaptación (McCubbin et al., 2002, I04; Patterson, 2020: 35 I). En la fase de ajuste, la familia hace pocos cambios, sigue los patrones de funcionamiento ya establecidos, utiliza los recursos y estrategias de afrontamiento habituales y resuelve los pequeños problemas. Si estos cambios no son suficientes y la familia entra en crisis (es decir, en un estado de desorganización y de necesidad de cambios), se pasa a la fase de adaptación. En ella, la familia es más vulnerable, ya que tiene que hacer frente a una serie de situaciones no habituales, provocadas por la enfermedad del niño, que abarcan múltiples ámbitos (familiar, laboral). Esta fase supone cambiar los patrones de funcionamiento, buscar nuevos recursos y apoyos, activar nuevas estrategias de afrontamiento y modificar las creencias, metas y valores de la familia.

Después de la fase de adaptación, los miembros de la familia y la familia en su conjunto son capaces de afrontar los retos de la enfermedad, trabajando juntos para desempeñar los roles y responsabilidades exigidos por ella (McCubbin et al., 2002, I04, Patterson, 2020: $35 \mathrm{I}$ ). Las crisis y las adversidades persistentes afectan a toda la familia. La respuesta que la familia dé a la enfermedad es crucial. La crisis es más aguda en las familias que tienen recursos limitados y concepciones muy negativas de la enfermedad, mientras que es menor cuando las familias disponen de recursos y tienen una concepción positiva.

Una enfermedad crónica es siempre una experiencia inesperada y traumática, y un elemento desestabilizador para cualquier familia. La enfermedad produce cambios en: a) las rutinas familiares (las comidas, la asignación de papeles, las divisiones del trabajo y las 
actividades recreativas o celebraciones festivas); b) los planes y prioridades de las familias, a los que dedica más recursos, tiempo y atención; y c) el modo en que se expresan las emociones (Grau y Espada, 20I2: I 26; Steinglass, I998: 55 ).

El diagnóstico, las intervenciones quirúrgicas, las reacciones adversas a los tratamientos y a sus secuelas, las recidivas, la muerte del propio hijo y la de otros niños, los cambios en su vida cotidiana, en la de sus hijos y en la de la familia extensa, la adaptación al hospital, la relación con los profesionales de la sanidad, el manejo de la información: todas son situaciones estresantes que los padres intentan solucionar controlando sus emociones negativas, desarrollando competencias en el manejo de la enfermedad, reestructurando sus valores y dando un nuevo sentido a su vida (Orbuck et al., 2005: I73).

Dos son las vivencias de los padres: a) un mundo que se desmorona (pérdida de sus puntos de referencia y del sentimiento de seguridad, cambios en la vida diaria, y dependencia de otros); y b) la lucha por sobrevivir (sentimientos de esperanza, centrarse en lo positivo, control para reducir el caos y no sentirse solos) [Björk et al., 2005: 269-270]. Los padres se enfrentan a diferentes situaciones estresantes relacionadas con (Patterson et al., 2005: 395):

- La naturaleza de la enfermedad y sus tratamientos: enfermedades secundarias a los tratamientos, pérdida de la integridad física y funcional, infertilidad, cirugía recurrente, déficits de atención y secuelas cognitivas.

- Las reacciones del hijo: miedo al tratamiento, pesadillas, consciencia de las reacciones de los otros (se siente diferente) e imposibilidad de mantener sus actividades habituales.

- Las reacciones y los problemas de la familia. Así, los padres pueden sentirse abrumados, impotentes, sin control, atemorizados por la posible muerte del hijo y por las recidivas, apenados y culpables; pueden tener incertidumbre sobre el futuro y pensamientos invasivos, no encontrar el equilibrio entre las múltiples necesidades de los miembros de la familia (sobreprotección/ independencia del niño, información al hijo y toma de medicamentos), tener conflictos con la familia extensa, temer la pérdida de la normalidad en la vida familiar y las reacciones de los otros hijos (ira, resentimiento, celos, exceso de responsabilidad), y tener problemas económicos y conyugales (formas diferentes de enfrentarse a la enfermedad, conflictos y falta de apoyo).

- La reacción del entorno social: distanciamiento por parte de las amistades de la familia y del niño enfermo, ausencia de apoyos de la Administración y de la escuela, la muerte de otros niños y la escasez de ayudas económicas.

- Las características del sistema sanitario: falta de conocimientos y de competencia de los profesionales, insensibilidad en la comunicación, retrasos en el diagnóstico y resultados de las pruebas, y precipitación en la toma de decisiones médicas.

Ante estas fuentes de tensión y estrés, la familia dispone de una serie de recursos psicológicos, personales y organizativos para afrontarlas (Patterson et al., 2005: 399):

- $\quad$ Capacidades y actitudes del niño enfermo: responsabilidad, madurez, capacidad para tolerar el dolor, actitud positiva y sentido del humor.

- Características de la familia: apoyo de la familia extensa, creencias religiosas, competencia y eficacia de los padres, comunicación abierta, apoyo de los otros hijos y sólida relación conyugal.

- Comunidad: apoyo de la comunidad religiosa, de las amistades, de las asociaciones y redes sociales de afectados, de la escuela y de los programas específicos.

- Sistema sanitario: médicos competentes y dedicados, apoyo del personal de 
enfermería y trabajadores sociales, horarios flexibles, y ayudas económicas.

Los factores que favorecen la resiliencia en las familias con enfermedades crónicas son: la rápida movilización y reorganización de la familia; el apoyo del equipo de cuidados sanitarios, de la familia extensa y de la comunidad; y los cambios de valores de la familia (McCubbin et al., 2002: 105). Gardner et al. (2006) han desarrollado un inventario (IFPF) para evaluar los factores protectores de la familia, siguiendo este enfoque.

\subsection{Resiliencia y procesos clave}

Desde una perspectiva más clínica, Walsh (I996) considera la resiliencia como los procesos interactivos que fortalecen al individuo y a la familia en el transcurso del tiempo. Tres son los procesos claves en la resiliencia familiar: el sistema de creencias de la familia, los patrones organizativos por los que se rige, y el tipo de comunicación y de resolución de los problemas. Estos procesos son los objetivos que deben regir cualquier intervención de los profesionales que quieran fomentar la resiliencia familiar (Walsh, I996: 8; 2002: I32; 2003: I7; Rolland y Walsh, 2006: 528). Caruso y Mikulic (2008) han desarrollado un Inventario para Evaluar el Potencial Resiliente Familiar (IPRF), en el que se miden los procesos claves.

\subsubsection{Sistemas de creencias}

Las familias más resilentes son aquellas que (Walsh, 2002: I32; Walsh, 2003: I7; Rolland y Walsh, 2006: 528):

- Encuentran sentido a la adversidad: normalizan y contextualizan los cambios y el estrés, considerando que éstos tienen un significado y que son comprensibles y manejables; y descubren las creencias y atribuciones sobre la causa de la enfermedad que generan tensiones, confunden la toma de decisiones y que bloquean la adaptación. Normalmente, estas creencias son el resultado de una combinación de información médica, atribuciones individuales y familiares.

- Adoptan una perspectiva positiva: la esperanza es el motor que las impulsa a actuar y a superar los obstáculos; el coraje y ánimo les permite afianzar las fortalezas y concentrarse en su potencial; y la iniciativa activa y perseverancia las ayudan a dominar la situación y provocar los cambios necesarios.

- Desarrollan valores de transcendencia $y$ espiritualidad como: las creencias, la fe, el apoyo de su comunidad religiosa y los rituales de curación; la inspiración, imaginando nuevas posibilidades, expresándolas de manera creativa y realizando acciones sociales; y la transformación, desarrollando la capacidad de aprender, de cambiar y de crecer como personas.

\subsubsection{Patrones organizativos}

Las familias con resiliencia son aquellas cuya organización tiene las siguientes características (Walsh, 2002: I32; 2003: I7; Rolland y Walsh, 2006: 528):

- Flexibilidad: son familias abiertas al cambio, lo que les permite reorganizase en función de las necesidades de la enfermedad; son capaces de mantener una estabilidad ante los cambios perturbadores, manteniendo la continuidad, la confianza y la perseverancia; y desarrollan un fuerte liderazgo en la crianza, protección y orientación de los hijos.

- Conectividad: son familias que se apoyan mutuamente, colaboran y están muy comprometidas; respetan las necesidades individuales, las diferencias y límites de cada miembro de la familia; y son capaces de reconciliarse y reconstruir las relaciones dañadas. 
- Disponen de recursos sociales y económicos: son familias capaces de movilizar los recursos familiares, sociales y de las redes comunitarias; saben buscar mentores y construir una seguridad económica; y conciliar la vida laboral con la familiar.

\subsubsection{Comunicación y resolución de problemas}

Otros aspectos clave en los proceso de resiliencia familiar son los relacionados con la comunicación y resolución de problemas. Así, las familias con resiliencia son capaces de (Walsh, 2002: I 32; Walsh, 2003: I7; Rolland y Walsh, 2006: 528; Herzer, 20I0: 28):

- Comunicarse con claridad: son congruentes entre lo que dicen y lo que hacen, y clarifican los mensajes ambiguos, buscando la verdad y diciéndola.

- Expresión emocional abierta: saben expresar sus sentimientos y participar de éstos (alegría y dolor, esperanzas y temores), tienen mutua empatía y tolerancia hacia las diferencias, son responsables de sus sentimientos y comportamientos y no se sienten culpables, disfrutan en la interacción, y tienen sentido del humor.

- Resuelven los problemas de forma colaborativa: son capaces de generar ideas creativas, aguzar el ingenio y aprovechar las oportunidades. Toman las decisiones de forma compartida, saben resolver conflictos, negociar y mantener la equidad y reciprocidad. Se centran en conseguir los objetivos, en tomar medidas concretas y en aprender de las dificultades. Tienen una actitud proactiva, previniendo los problemas, evitando las crisis, y preparando los cambios futuros.

\subsection{Sistema familiar y enfermedad}

Desde este enfoque (The Family System-Illness, FSI), Rolland (I994) estudia la resiliencia familiar en el contexto de las enfermedades crónicas.
Abarca tres dimensiones: a) las características de la enfermedad; b) las fases de la enfermedad; y c) el ciclo vital individual y familiar, y las variables clave en el sistema familiar (creencias, cultura, etnia, espiritualidad y género).

\subsubsection{Características de la enfermedad}

Las características de la enfermedad influyen en la forma de reaccionar de las familias. Cada enfermedad tiene sus propias características y exigencias. Las reacciones son diferentes en función de que sea aguda o gradual; de que su curso sea progresivo, constante o con recaídas; de que su resultado suponga un acortamiento de la vida o la muerte; de que derive en discapacidad o no; y de que esté en la fase de crisis, crónica o terminal (Rolland y Walsh, 2006: 529; Grau y Fernández, 20I0: 206; Herzer et al., 2010: 29):

- Un comienzo agudo de la enfermedad hace que la familia tenga menos tiempo para adaptarse, y conlleva un mayor desgaste, al tener que compatibilizar el mantenimiento de su propia identidad con las demandas de la enfermedad.

- Un comienzo gradual permite a la familia disponer de más tiempo para adaptarse y una pérdida menor de la propia identidad (Rolland y Walsh, 2006: 529; Grau y Fernández, 2010: 206).

- Un curso progresivo de la enfermedad requiere que la familia esté en una adaptación continua, por lo que los momentos de respiro son menores.

- En las enfermedades de curso constante, el niño se recupera en mayor o menor medida; la enfermedad se estabiliza, pero siempre queda un déficit o limitación que es asumido por la familia o el cuidador primario.

- Cuando cursa con recaídas, la familia pasa por periodos de remisión de los síntomas durante los cuales desarrolla sus rutinas, y 
periodos de aumento de los éstos, viviendo con estrés la posibilidad de una recaída.

- $\quad$ En las enfermedades que amenazan la existencia, puede que la adaptación pase por un 'duelo anticipado', aislando al enfermo y apartándolo de ciertas tareas o actividades. La incertidumbre puede llevar a la sobreprotección.

- El grado de incapacidad que provoca la enfermedad implica ajustes en la adaptación relacionados con el comienzo, curso y resultado. Si es progresiva, la incapacitación mayor ocurre en las últimas fases, lo que permite a las familias anticipar recursos y estrategias (Rolland y Walsh, 2006: 530; Grau y Fernández, 2010: 206).

\subsubsection{Fases de la enfermedad}

La fase de crisis corresponde al periodo previo al diagnóstico, en la que ya se están dando los síntomas y existe la sospecha de que algo no va bien. En este periodo hay que aprender a convivir con los síntomas, adaptarse al hospital, dar significado a la enfermedad, aceptar el cambio, reorganizarse y colaborar con el equipo médico.

La fase crónica es intermedia entre el diagnóstico y la problemática que envuelve la fase terminal. Hay que compatibilizar las necesidades del niño con las de la familia, maximizar la autonomía de todos los miembros, prever el posible impacto de las fases futuras, vivir con incertidumbre y mantener una comunicación abierta.

En la fase terminal, la sensación de pérdida está presente. Hay que afrontar la muerte y trabajar el duelo (Rolland y Walsh, 2006: 53 I; Grau y Fernández, 20I0: 206).

El diagnóstico y la hospitalización son las primeras situaciones críticas con las que tiene que enfrentarse la familia: el hospital, la separación del hogar, dejar a los demás hijos al cuidado de otras personas y e interrumpir las actividades habituales de la familia. La presencia de los padres en el hospital y la participación en los cuidados y otras actividades hospitalarias ayudan a mantener y fortalecer las relaciones entre los padres y el niño enfermo, y asimismo les proporciona a los padres un sentimiento de dominio de la situación (Grau y Fernández, 2010: 2004).

Los tratamientos y las secuelas también son una fuente de estrés. Cuando una enfermedad ha remitido, lo que más preocupa a los padres es la reaparición. En el caso de las recidivas, toda la familia sufre enormemente y su adaptación es más compleja cuanto más largo ha sido el periodo de remisión. Cuando hay secuelas graves, éstas se convierten en el centro de sus vidas y de sus necesidades económicas, y pueden ser el desencadenante de conflictos familiares (Grau y Fernández, 2010: 205).

La muerte del hijo implica la reorganización familiar a largo y corto plazo. La familia tiene que pasar el duelo, y para ello tiene que reconocer la pérdida, compartir el dolor, reorganizar el sistema familiar sin el ser querido, y establecer nuevas relaciones y metas (Grau y Fernández, 2010: 205).

\subsubsection{Ciclo vital individual y familiar, y variables clave en el sistema familiar}

Además de las características y fases de la enfermedad, el modelo también se centra en: la interacción de la enfermedad con el desarrollo individual y con el de la familia; la historia de cómo las familias han afrontado las enfermedades a lo largo de las generaciones; el sistema de creencias familiares respecto a la salud/enfermedad y el significado que dan a las condiciones del niño enfermo; los recursos familiares, sociales y de la comunidad disponibles para gestionar las crisis médicas y el cuidado de la enfermedad a largo plazo; y las relaciones entre las instituciones sanitarias, los profesionales, el niño enfermo y la familia (Rolland y Walsh, 2006: 533 ).

\subsection{Ecológico y de desarrollo}

Desde los enfoques ecológico y de desarrollo (Falicov, I995, 2007; McGoldrick, 2005; 
Masten, 2009), se considera que la resiliencia, los modos de ver el mundo y los comportamientos adaptativos de la familia dependen de la pertenencia simultánea y su participación en múltiples contextos: el hábitat (rural, urbano o suburbano), la lengua, la edad, el género, la configuración familiar, la raza, la etnia, la religión, la nacionalidad, el estatus socioeconómico, el empleo, la educación, la ocupación, la orientación sexual, la ideología política, la migración y los estadios de aculturación, las transiciones del ciclo vital familiar, la etapa evolutiva de los niños, el grupo de amigos, los apoyos sociales, la escuela, los servicios sanitarios y otros sistemas (Falicov, 2005: 2, 7; McGoldrick, 2005: I6-I7; Walsh, I996: 264; 2003: 3; Villalba, 2003: 293).

\subsubsection{Ecológico}

La familia se desenvuelve en un contexto ecológico y establece relaciones en función de su comunidad (racial, étnica, religiosa, rural/ urbana, redes sociales), del lugar donde vive (vivienda, barrio y seguridad del barrio), del trabajo (ingresos, estabilidad, horarios, satisfacción y discriminación), de las escuelas (rendimiento, implicación de los padres, disciplina, raza/etnia) y de otras instituciones (sistemas legales y sanitarios) [Falicov, I995: 5].

Asimismo, en una sociedad multicultural y globalizada, la familia depende de los procesos de migración/aculturación. Los procesos de desarraigo cultural pueden ser internos (separación y reunión, trauma y crisis, dolor y duelo, desorientación/ansiedad e identidad cultural) o externos (lenguaje, redes sociales, instituciones, valores) [Falicov, I 995: 5; 2007: I 59; McGoldrick et al., 2005: I].

La familia depende del desarrollo de su propio ciclo vital (normas e ideales, estadios, contenido apropiado a la edad, sincronización de secuencias, mecanismos de cambio, sincronización de rituales y ritos) y de su organización: características de las diadas marido/mujer y padres/hijos (claras y exclusivas o claras e inclusivas, igualitarias o jerárquicas, directas o indirectas, individuales o conectadas, coalición intergeneracional o en la misma generación), de los límites (próximos o jerárquicos), del estilo de comunicación, de los valores y de las amenazas (Falicov, I995: 5).

La reacción de la familia a las enfermedades depende de la cultura: por ejemplo, los padres chinos no muestran algunos de los sentimientos de los occidentales, como la culpa o depresión. Los padres chinos aceptan la enfermedad como su destino; también sus creencias activan pensamientos positivos y raras veces muestran el amor a su hijo (el celo en la preparación de los alimentos simboliza la expresión de su amor y preocupación); no suelen darle información y su cuidado es lo prioritario (Wong y Chan, 2006: 7I4). Aunque hay similitudes entre los padres de diversas culturas, es conveniente respetar la singularidad de las familias y su cultura.

Las reacciones también dependen del género. Existen bastantes estudios sobre las reacciones de las madres hacia la enfermedad del hijo y, en menor medida, sobre las de los padres. Las madres se implican más en la enfermedad, mientras que los padres participan, pero no se implican. Asimismo, los padres controlan sus emociones, porque consideran que son un signo de debilidad o porque no quieren revelarlas, y pueden ser menos empáticos para entender el estilo de afrontamiento de cada uno de los miembros de la familia (Brody y Simmons, 2007: I 52, I 53 ).

Los hijos tienen expectativas diferentes respecto a los comportamientos y actitudes del padre y de la madre. A la madre se les asocia a la crianza y a la comprensión, mientras que al padre se le percibe como poderoso y digno de respeto, aunque estos roles pueden variar de una cultura a otra. Las relaciones entre el padre y la madre con el hijo y su calidad de vida dependen del género de sus progenitores (Orbuck et al., 2005: I73).

Normalmente, las madres asumen el papel de cuidador primario, que se convierte en el eje de la familia, pero también en el miembro más vulnerable. Habitualmente, esta adscripción 
sigue criterios socioculturales que asigna esta función a la mujer. El bienestar de ésta depende de la gravedad de los síntomas del enfermo, de los apoyos sociales de que disponga, y de la percepción que tiene de la carga que le supone y de los beneficios que obtiene. Necesita apoyo y desarrollar competencias (Fernández et al., 20I2: 299; Grau y Fernández, 2010: 207).

Un estatus socioeconómico bajo también puede tener consecuencias negativas en el funcionamiento familiar, y agudizar los conflictos de pareja y el estrés.

Los apoyos sociales del equipo de salud (información, apoyo emocional y participación en los tratamientos), de la familia extensa (apoyo emocional e instrumental), de la comunidad (amigos, asociaciones), del trabajo (flexibilidad en los horarios, capacidad de ausentarse y retornar) son fundamentales para el desarrollo de la resiliencia familiar (Brody y Simmons, 2007: I 54, I 55 ).

\subsubsection{De desarrollo}

La resiliencia familiar depende no sólo de su evolución multigeneracional, sino también de su propio ciclo vital y del de los miembros que la componen (Carter y McGoldrick, 2005: 3). Así, es muy importante describir cómo han surgido los cambios en la familia y qué respuestas se han dado, cuántos factores estresantes (internos y externos) ha soportado la familia y cómo ésta ha resuelto las crisis en las sucesivas generaciones (Walsh, 2003: 4). Las respuestas de la familia ante una crisis están fuertemente influenciadas por su historia (Carter y McGoldrick, 2005: 3 ). Las enfermedades que la familia ha sufrido en generaciones anteriores, el modo como se ha enfrentado a ellas y los cambios organizacionales que se han producido influyen en las expectativas futuras de la familia, y en la visión optimista o catastrofista que ésta tiene de la enfermedad. Estos modelos de comportamiento se transmiten a la largo de las generaciones y afectan a su sistema de creencias (de lo que se enorgullecen, de sus vergüenzas, mitos, tabús y expectativas). Una evaluación multigeneracional (a través de genogramas) puede clarificar las áreas más vulnerables de cada familia y detectar a las familias con alto riesgo, y, desde un enfoque orientado a la resiliencia, puede determinar las influencias positivas del pasado en el presente y en el futuro. Esta información es muy importante para comprender el significado de cómo la familia vive la enfermedad, predecir el estilo de afrontamiento, ajustar los cambios necesarios en el futuro y planificar la intervención (Rolland y Walsh, 2006: 533).

Asimismo, una enfermedad crónica pediátrica influye en el desarrollo del niño y de los otros miembros de la familia dependiendo de la edad en que se contrae la enfermedad. Una perspectiva basada en el ciclo vital nos facilita un pensamiento proactivo acerca de la duración y naturaleza de las tensiones en la familia en su conjunto y en cada miembro en particular (Rolland and Walsh, 2006: 534).

En el caso de las enfermedades crónicas pediátricas, el ciclo vital de la familia corresponde a la etapa de crianza de los niños o adolescentes; sin embargo, en nuestra sociedad los cambios en la familia tradicional son muy rápidos y nos encontramos con una gran diversidad de familias. Muchos niños viven en más de un hogar, en el caso de padres divorciados o que se han vuelto a casar. Pueden vivir con un único padre divorciado, con familias que se han vuelto a casar o con los hijos de la pareja de uno de sus padres, con progenitores lesbianas o gais, con un único padre soltero y con parejas de hecho. Las intervenciones familiares deben tener en cuenta esta amplia variedad de familias (Carter y McGroldrick, 2005: IO)

Normalmente la enfermedad fuerza un momento centrípeto de transición en la familia para adaptarse a las necesidades que aquélla impone. Los momentos centrípetos son aquellos en los que la familia tiene que realizar tareas que son internas a ella. Si la familia, en el momento en que se produce una enfermedad, se encuentra en un momento centrípeto, éste se alargará en el tiempo. Si coincide con un momento centrífugo (la familia desarrolla tareas externas a sí misma), 
puede sacar a la familia de su ciclo natural (Grau y Fernández, 20I0: 208).

Asimismo, la enfermedad afecta al desarrollo físico, cognitivo, emocional y social del niño y adolescente enfermo. Al niño, la enfermedad le crea una dependencia respecto al sistema sanitario, grandes preocupaciones por su vida y bienestar, tensión emocional, disrupciones en las relaciones familiares, estigma social y alienación, y cambios importantes en las rutinas familiares (Osbruck et al., 2005: I73). Por ejemplo, en niños pequeños enfermos de cáncer, las secuelas neurocognitivas de los tratamientos son más importantes, por lo que las dificultades de aprendizaje serán mayores, sobre todo en una etapa en la que el niño tiene que aprender las habilidades básicas de lectoescritura y matemáticas. Por el contrario, en la adolescencia los cambios en su apariencia física pueden provocarle desequilibrios y desajustes psicológicos, así como también el incremento de la dependencia respecto de los cuidadores, precisamente en una etapa en la que se inicia un proceso de autonomía (Grau, 20I2: I78). En casa se le somete a una disciplina que no corresponde con su edad, lo que genera conflictos, al tener que negociar la autonomía ya conseguida en etapas anteriores (Grau y Fernández, 20I0: 207).

Todos los enfoques sobre resiliencia en niños y jóvenes coinciden en que ésta constituye un conjunto de atributos individuales, de relación y contextuales, asociado a un buen ajuste y desarrollo bajo una variedad de condiciones amenazantes en un contexto cultural y a lo largo del ciclo vital. Entre los factores protectores de la resiliencia en niños y jóvenes, se señalan (Masten et al., 2009: I 26, I27):

- $\quad$ En el niño: capacidad para resolver problemas, para autocontrolarse, temperamento fácil en la infancia y personalidad adaptable a lo largo del desarrollo, autopercepciones positivas o autoeficacia, fe y sentido a la vida, una visión optimista de la vida, talento reconocido por él mismo y por la sociedad, y capacidad de atracción.
- En la familia: relaciones positivas de apego; relaciones estrechas competentes, prosociales y de apoyo con los adultos; autoridad paterna (calidez y expectativas); clima positivo entre los padres, con pocas discusiones; entornos familiares estructurados; estudios postsecundarios en los padres; padres con cualidades para desarrollar factores protectores e implicados en la educación de sus hijos, con un buen estatus socioeconómico, respetuosos con las normas; y padres con relación romántica con modelos prosociales y de bienestar.

- En la comunidad y en las relaciones con las organizaciones: escuelas eficaces, altos niveles de seguridad pública, barrios con alta eficacia colectiva, buenos servicios de urgencia y de salud.

Trabajar bajo las premisas de la resiliencia supone priorizar objetivos positivos, promocionar el desarrollo saludable y competente, y prevenir los problemas con la misma finalidad. Entre las estrategias para fomentar la resiliencia, se pueden señalar: estrategias centradas en el riesgo, que previenen riesgos y estresores; estrategias centradas en las fortalezas, que mejoran el número y la cantidad de recursos o capital social; y estrategias centradas en el proceso, que movilizan el poder de los sistemas de adaptación humana (Masten et al., 2009: I28).

\section{Principios de intervención}

Todos los enfoques estudiados tienen una repercusión práctica en la mejora de la resiliencia familiar, y para fomentarla es imprescindible:

- Conocer si la familia está en una fase de ajuste o de adaptación, y considerar que las crisis familiares producidas por la enfermedad no tienen un significado peyorativo, sino que suponen una situación 
de desorganización y de necesidad de cambio. Es imprescindible conocer cuáles son los factores protectores (recursos psicológicos, personales y organizativos) de la familia para afrontar las fases de ajuste y adaptación, y para gestionar el cuidado de la enfermedad a largo plazo, con el objeto de fortalecerlos.

- Tener una visión clara de los objetivos y finalidades de la intervención, promoviendo aquellos procesos que favorecen la resiliencia, referentes al sistema de creencias de la familia, a los patrones organizativos por los que se rige, y al tipo de comunicación y de resolución de los problemas.

- Contextualizar estos procesos según las características y fases de la enfermedad.

- Evaluar los contextos en los que la familia habita, con el objeto de comprender los desafíos, recursos y limitaciones.

- Conocer cómo incide la enfermedad en el desarrollo individual del niño y en el de la familia.

- Conocer cómo las familias han afrontado las enfermedades a lo largo de las generaciones; el sistema de creencias familiares respecto a la salud/enfermedad; y el significado que dan a las condiciones del niño enfermo.

- Conocer las relaciones entre las instituciones sanitarias, los profesionales, el niño enfermo y la familia.

Asimismo, es imprescindible respetar la singularidad de las familias, tener una comprensión multidimensional del problema, y disponer de servicios centrados en la familia y sus necesidades, integrados y coordinados (Grau y Fernández, 20I0: 209-2II):

\subsection{Singularidad de las familias y comprensión multidimensional}

Cada familia reacciona y vive las crisis de manera diferente y propia (Falicov, I995:
I). Todas las familias pasan por las mismas fases, pero sus reacciones y acciones son diferentes. La familia suele desarrollar sus propias estrategias como una respuesta normal a un sistema desequilibrado, provocado por la enfermedad. Las reacciones de la familia dependen de numerosos factores: experiencia en situaciones de crisis y problemas médicos, estatus socioeconómico, cultura, nivel de conocimientos, calidad de los servicios sanitarios y sistemas de apoyo; y no suelen ser patológicas. Cada familia es una unidad diferenciada, con antecedentes socioculturales, experiencias y recursos propios (Falicov, I995: 5). Este enfoque multidimensional nos permite comprender el sentido que cada familia otorga a la vida antes y después de la enfermedad. Las familias necesitan ser comprendidas y no interpretadas. Sus valores culturales deben ser respetados y no cuestionados. Asimismo, este enfoque nos explica por qué algunos niños y sus familias son más resilientes o más vulnerables ante la enfermedad y sus secuelas (Grau, Fernández, 20IO: 209; Borges y Silva, 2009: 25 I-252).

\subsection{Servicios centrados en la familia}

De los diferentes modelos de programas orientados a los padres (centrados en los profesionales, la familia como aliada, enfocados a la familia y centrados en la familia), el modelo más idóneo para el fomento de la resiliencia es el centrado en la familia. Este modelo considera que las familias son capaces de tomar decisiones informadas y actuar en consecuencia (Dunst et al., 201 2: 223).

Los profesionales fortalecen las estructuras existentes y promueven la adquisición de nuevas habilidades. Sus intervenciones se centran en el fomento de las capacidades y en la movilización de recursos y apoyos. Asimismo, utilizan estrategias de ayuda relacionales (empatía, calidez, autenticidad, confianza en las capacidades de la familia), y participativas que reconocen la responsabilidad de la familia en la búsqueda de soluciones a sus problemas y en la adquisición de los conocimientos y de las destrezas en función de las circunstancias (Dunst et al., 20I 2: 222). 
Se enfatiza la capacidad de la familia para resolver sus problemas, ya que dispone de las competencias necesarias para efectuar los cambios necesarios (Borges y Silva, 2009: 252). Así, es la familia la que asume la responsabilidad del manejo diario del enfermo y es la que mejor nos puede enseñar acerca del impacto que la enfermedad crónica tiene en su vida, y de las actitudes y conductas que son más útiles para superar dicho impacto.

Los profesionales, más que intervenir, deben acompañar y ayudar a las familias a darle significado a esta situación, ya que éstas tienen experiencias equivalentes a las de ellos en el tratamiento de la enfermedad (Grau y Fernández, 2010: 209). La evaluación de su problemática debe centrarse en cómo sus miembros juzgan los acontecimientos estresantes y en qué estrategias han utilizado para superar los problemas. Es más importante evaluar las prioridades y necesidades de las familias que realizar evaluaciones clínicas. La intervención debe ser acorde con los objetivos de cada familia. Sus aspiraciones después de la enfermedad siguen siendo las mismas que las de todas las familias, y su interpretación depende de cada una. La familia debe mantener su identidad frente a la enfermedad y los profesionales deben estar preparados para entender sus puntos de vista (Grau y Fernández, 2010: 209).

\subsection{Servicios centrados en las necesidades de los consumidores}

Los servicios deben organizarse en función de las necesidades de los usuarios (enfermos y familias), y no de los profesionales. Las necesidades de una familia pueden concretarse en las que tienen un impacto en la merma de la calidad de vida y las que tienen relación con el papel que desempeña aquélla en la mejora de la calidad de vida del niño enfermo (Freixas, 200I: I49).

En el primer supuesto, la familia es receptora de servicios; en el segundo, es proveedora. Cuando es receptora, puede participar en servicios de respiro familiar, de orientación, de apoyo (grupos de autoayuda de padres y familiares), de terapia familiar y de redes sociales (asociaciones)
[Freixas, 200I: I49]. Cuando es proveedora de servicios, participa en el tratamiento del hijo (hospitalización domiciliaria) y en las actividades formativas. Las familias deben compartir responsabilidades con los profesionales, y ser parte activa en la toma de decisiones y en su implementación, ya que el control de la enfermedad permite que el niño tenga una mejor calidad de vida, evita los reingresos hospitalarios, normaliza las relaciones sociales y favorece la integración escolar y social.

Se debe conseguir que la familia sea capaz de gestionar y dirigir los cuidados necesarios al niño enfermo, apoyándose en los recursos disponibles en su entorno social (voluntariado, asociaciones de afectados, de ocio, vecinales). Hay que ser creativos y medir el coste/eficacia de los programas de entrenamiento.

\subsection{Servicios integrados y coordinados}

La coordinación debe entenderse como un proceso colaborativo de análisis de los servicios disponibles, de planificación, de puesta en práctica, de coordinación y de evaluación de la atención proporcionada al niño enfermo y a su familia, y no como la mera organización del uso de los servicios asistenciales (Grau y Fernández, 20I0: 209). La colaboración interdisciplinar y la posibilidad de integrar muchos servicios de disciplinas dispares dentro de un conjunto de intervenciones tienen un considerable valor funcional para los niños y familias (Guralnick, 2005: 5).

\section{Estrategias de intervención}

En una enfermedad crónica, intervienen un conjunto de profesionales provenientes de diferentes ámbitos (sanidad, educación, trabajo social), a los que se les puede añadir la de instituciones relacionadas con la sociedad civil. Los padres y el niño enfermo interactúan con profesionales, y desde su ámbito de actuación pueden fomentar la resiliencia familiar, que será 
un factor importante en la calidad de la familia en su conjunto. A continuación, vamos a señalar algunas estrategias que pueden desarrollar estos profesionales para favorecer la resiliencia familiar.

\subsection{Sistema sanitario: médicos}

Con el diagnóstico de una enfermedad crónica pediátrica, se inicia un largo camino en las relaciones entre los médicos, los padres y los hijos enfermos, que son decisivas para una adecuada adaptación a las exigencias de la enfermedad (Grau et al., 2010, 278). La relación médico-niño-padres es fundamental para el tratamiento de una enfermedad crónica $\mathrm{y}$, cuando no es satisfactoria, suele generar conflictos, dificultades para mantener la adherencia terapéutica, abandono o exceso terapéutico. Los médicos deben tener una comunicación fluida con los padres y con el niño enfermo, darles apoyo emocional e instrumental, desarrollar sus competencias para el manejo de la enfermedad y colaborar con los profesionales de otros subsistemas (Grau et al., 2010: 278; Medina-Sanson et al., 2006: 289).

Los médicos son la principal fuente de información de los padres: les comunican el diagnóstico, los resultados de las pruebas, los tratamientos a los que van a ser sometidos su hijo, las secuelas de los tratamientos y de la enfermedad, las posibles recidivas o la inmediatez de la muerte del niño. Los psicólogos pueden ayudar a los médicos en los procesos de información a los padres y niños enfermos (Gaite et al., 2008: I70; Grau et al., 2010: 278). También pueden los médicos informarles y aconsejarles acerca de cómo convivir con la enfermedad; cómo continuar con una vida familiar, social y laboral satisfactoria; de qué recursos sanitarios y asistenciales se dispone; y cómo participar activamente en el tratamiento. Deben resolver las dudas que surgen y dar consejos sobre los cuidados que el niño precisa, sobre cómo normalizar la vida del niño y sobre cómo deben comportarse para que este objetivo sea cumplido (Grau et al., 2010: 278).
La relación no es únicamente instrumental, sino también emocional. La comunicación entre los médicos y padres va más allá de la mera transmisión de datos técnicos y pronósticos del proceso terapéutico: depende de la relación que se establece con el niño y con su familia, y de su colaboración en el tratamiento. Siempre debe ser "a la medida" teniendo en cuenta circunstancias y edad (Prado et al., 2007: I97). El médico debe ser capaz de crear un ambiente de confianza y seguridad en el niño y en su familia, de escucharlos, y de favorecer la expresión de sentimientos y de emociones, y la toma de decisiones de forma compartida (Cruz et al., 2004: I22). La vivencia de la enfermedad del niño y de su familia es distinta a la del médico, y a su vez, cada niño y cada familia viven el sufrimiento de forma singular (Grau et al., 20I0: 278). Además, hay que tener en cuenta la diversidad cultural de las familias (Álvarez et al., 2008: 240).

Los médicos también deben potenciar la participación de los padres en el tratamiento, ya que ésta permite la reducción de los tiempos de hospitalización del niño, favorece la hospitalización domiciliaria y, en última instancia, mejora la calidad de vida del niño. Esto implica desarrollar procesos educativos protocolizados, adecuados a la enfermedad y a la capacidad de cada familia, e impartidos por los profesionales que se encargan de la atención integral del niño. Su objetivo fundamental es que los padres puedan autoayudarse y asumir responsabilidades (Díaz, I999: I92). En esta tarea es fundamental la ayuda del personal de enfermería. Asimismo, es imprescindible educar a los niños para el control de la enfermedad, el manejo de aparatos, la preparación para las pruebas diagnósticas y los tratamientos. Este objetivo se irá incorporando a medida que el niño crece y debe adaptarse a su nivel de desarrollo (Grau, 2004: 27).

Por último, los médicos realizan una función administrativa no sólo en la cumplimentación de las historias clínicas y los consentimientos informados, sino también en la elaboración de informes y certificados que les exigen a los padres en los servicios sanitarios (médico 
de cabecera), educativos (informes para los maestros y escuela) y sociales (ayudas). Esta actividad es de gran relevancia para la mejora de la calidad de vida de los niños enfermos y sus familias (Grau et al., 2010: 278).

\subsection{Sistema educativo: maestros}

El sistema educativo y los maestros de las aulas hospitalarias, de los servicios de atención educativa domiciliaria y de los centros educativos de referencia del niño enfermo tienen un papel fundamental en el desarrollo de la resiliencia familiar, ya que favorecen la continuidad escolar, reducen el absentismo escolar y normalizan su vida. Estos servicios deben ser coordinados también desde la escuela, para que el niño pueda incorporarse al centro escolar y favorecer su inclusión. Asimismo, los maestros deben dar una respuesta educativa a las necesidades educativas especiales que presentan estos alumnos, para lo que es imprescindible su colaboración y coordinación (Grau, 2012: 176).

La diversidad de las enfermedades crónicas pediátricas hace que las necesidades educativas especiales de estos niños sean muy heterogéneas, diversas, inestables, cambiantes y menos predecibles que las que presentan otros alumnos. Es imprescindible trabajar en tres ámbitos diferentes: conseguir el control de la enfermedad por el niño y adaptar la escuela para ofrecer los cuidados que precisa; ayudar al niño a expresar sus emociones y sensibilizar a los compañeros sobre la enfermedad y sus consecuencias; y hacer las adaptaciones curriculares necesarias para compensar las secuelas cognitivas, sensoriales, motoras y afectivas derivadas de la enfermedad y de los tratamientos (Grau, 2004: 25).

Los maestros y la escuela deben tener información referente a las características de la enfermedad y de los tratamientos, secuelas y cuidados que precisa el niño; y adoptar las medidas necesarias para su control. La adaptación del entorno escolar le permite al niño normalizar sus relaciones sociales, manteniendo, a su vez, los cuidados que precisa (Grau, 2004: 3I).
El maestro también tiene que tener en cuenta los problemas emocionales no sólo del niño enfermo, sino también de sus hermanos. Entre las estrategias que puede utilizar para paliar estos efectos, podemos señalar: animar al niño $\mathrm{y}$ a sus hermanos a expresar sus sentimientos, desviar su ira hacia otras actividades, mantener el contacto con los amigos y compañeros de clase, favorecer las amistades en el patio, reforzar la toma de decisiones, evitar los gimoteos y los lloros, darle información sobre la enfermedad, y que el niño participe activamente en algún proyecto de clase (Grau, 2004: 49).

Respecto a los compañeros de clase, los maestros deben informarles sobre la situación del niño y aspectos de su enfermedad, prever las posibles burlas o bromas respecto a su apariencia física, sugerirles pautas sencillas de comportamiento hacia su compañero -ayudar, pero no compadecer; interesarse por su enfermedad, pero no interrogar; y ser amables, pero no complacientes o consentidores-, y preparar una pequeña bienvenida cuando el niño regresa a la escuela después de la hospitalización (Grau, 2004: 78).

Respecto al niño enfermo, los maestros deben ayudarle a superar situaciones cotidianas que pueden ser conflictivas para conseguir la normalización, no destacándole excesivamente de sus compañeros, exigiéndole como los demás siempre que sea posible, integrándole en grupos ya formados, y ofreciéndole seguridad y apoyo en los momentos difíciles en su relación con los compañeros (Grau, 2004: 78).

Respecto al currículo, el profesor debe adaptar la metodología, recortar y adaptar los contenidos, proporcionarle materiales de apoyo y flexibilizar los horarios escolares (Grau, 2004: I76). Cuando existen secuelas neuropsicológicas que dificultan el aprendizaje escolar, el equipo docente, con la participación de los maestros de educación especial y de audición y lenguaje, debe diseñar y poner en práctica, junto con el equipo psicopedagógico, las adaptaciones curriculares en función de las necesidades educativas de cada niño (Grau, 20I2: I76). 
En el contexto escolar, debe realizarse la evaluación psicopedagógica y las adaptaciones de acceso e individualizas; aplicar estrategias educativas individualizadas; cambiar la configuración de la clase, del currículo, de los objetivos de aprendizaje y de la evaluación; favorecer la socialización del niño; conocer los efectos tardíos de la enfermedad y de su tratamiento; incorporar las estrategias de intervención especializadas; y proporcionar los apoyos necesarios (Grau, 201 2: 176, I77).

Los maestros deben colaborar con las familias, desarrollar las competencias de éstas y considerarlas como un recurso imprescindible en la recuperación del niño. Las familias competentes colaboran activamente con los maestros en la educación de sus hijos, utilizan los servicios disponibles en la comunidad y son un apoyo importante para el niño (Grau y Fernández, 20I0: 2II).

Los maestros también deben colaborar con las asociaciones, el voluntariado, los servicios sociales y con los servicios sanitarios especializados, para mejorar la calidad de vida de niño (Grau, 2I 2: I77).

En última instancia, se trata de tener una mirada positiva y desarrollar las competencias del niño a pesar de la enfermedad, creando un clima de confianza, aceptando al niño como es, favoreciendo su participación en las distintas actividades, proporcionándole los medios necesarios, y transformar así las dificultades en una situación de aprendizaje. Para ello, los maestros deben establecer una relación personal, descubrir los aspectos positivos, ser empáticos, evitar la humillación, el menosprecio y la indiferencia, adoptar actitudes de respeto, solidaridad y comprensión, saber escuchar y poner límites (Muñoz y Sotelo, 2005: I I9).

\subsection{Psicólogos clínicos}

Los psicólogos pueden fomentar la resiliencia familiar, ayudando a las familias a buscar sentido a la enfermedad, a normalizar y contextualizar los cambios y la angustia, a desarrollar un enfoque positivo, a fortalecer el sentimiento de dominio sobre la enfermedad, a desarrollar sus competencias y a reestructurar sus valores y creencias (Rolland y Walsh, 2006: 535, 536).

Para influir en el sistema de creencias de la familia, se puede utilizar la técnica de la deconstrucción, que consiste en crear, conjuntamente con la familia, versiones de la realidad más adaptativas, para que sean capaces de dar sentido a la enfermedad y de convertir la desesperación en esperanza (Presbury et al., 2008: I25). Pueden utilizar la inmediatez (centrarse en lo ocurre en ese momento) para poner de relieve la dinámica organizacional de la familia y construir los potenciales de la familia (Presbury et al., 2008: 204). Asimismo, para establecer nuevos modelos de comunicación, pueden utilizar: a) la escucha de forma comprometida, y acogedora; b) la comprensión, repitiendo y parafraseando algunos aspectos y haciendo preguntas para clarificar; y c) la validación, asintiendo con la cabeza, sonriendo cálidamente y absteniéndose de sembrar dudas o de debatir con ellos (Presbury et al., 2008: 37-38).

La intervención puede desarrollarse de forma individual, en pareja, en la familia nuclear o extensa, y en grupos formados por padres, por niños enfermos o por varias familias (Rolland y Walsh, 2006: 529). Asimismo, los psicólogos pueden desempeñar sus funciones en el sistema sanitario, educativo o en el ámbito de las asociaciones.

\subsection{Otros profesionales y asociaciones}

Además de estos profesionales, los trabajadores sociales y las asociaciones de afectados pueden tener también un papel importante en el fomento de la resiliencia familiar.

Los trabajadores sociales pueden proporcionar información sobre los ayudas disponibles -certificados de minusvalías, prótesis, dietas para la hospitalización, servicios de ambulanciay gestionar su solicitud. Con su actuación, pueden mejorar la conciliación de la vida 
familiar y laboral, y los recursos económicos de las familias. Estos profesionales pueden actuar en el sistema sanitario, en la asistencia social estatal y autonómica, en ayuntamientos y en asociaciones de afectados.

Las asociaciones de afectados pueden ofrecer apoyo emocional e instrumental a las familias y niños afectados, al subsanar las deficiencias de los servicios públicos y reivindicar su implantación; favorecer la comunicación con otras familias y con otros afectados, para buscar soluciones a su problemática; desarrollar grupos de apoyo y de duelo; proporcionar ayudas económicas; e informar y sensibilizar a la sociedad.

\section{Conclusiones}

La resiliencia familiar es un concepto que se basa en las fortalezas de la familia, incluyendo la relación parental, de pareja, entre hermanos y de familia extensa. En las familias con resiliencia, a pesar de las adversidades, sus relaciones se enriquecen, sus miembros se aprecian más y son capaces de resolver los problemas de manera eficaz (Rolland y Walsh, 2006: 528).

El concepto de resiliencia ayuda a entender las complejas interacciones entre enfermedad biológica, la unidad familiar, los miembros de la familia y los profesionales involucrados en su atención; también los cambios importantes que surgen durante la enfermedad tanto en el individuo como en la dinámica familiar, y cómo sus creencias, sus valores y su legado multigeneracional influyen en la construcción de los significados de los problemas de salud y de las relaciones que se establecen con los profesionales (Rolland y Walsh, 2006: 528, 529)

Cada familia es un caso único, y puede ser ayudada por los diferentes profesionales para prever los cambios y conocer los problemas prácticos y emocionales que surgirán a lo largo de la enfermedad. Es imprescindible que los profesionales conozcan cómo afecta la enfermedad a las familias, que sean receptivos a sus preocupaciones y necesidades, y que faciliten el desarrollo de estrategias de afrontamiento que permitan a los padres solucionar los problemas, buscar información válida y apoyo profesional, reestructurar sus valores dando sentido a la enfermedad, controlar sus emociones, y buscar apoyo emocional en su familia y amigos. Estos profesionales deben ayudar a las familias a conseguir los procesos claves de la resiliencia, colaborando con aquélla.

La familia con resiliencia sabe manejar adecuadamente la enfermedad y proporcionar los cuidados que precisa el hijo enfermo; participa activamente en el tratamiento tanto en el hospital como en el hogar; busca información adecuada y la proporciona a sus hijos; controla los sentimientos negativos y la ansiedad; tiene una actitud positiva respecto a la resolución de problemas, evitando caer en la depresión; ayuda a sus hijos a controlar las emociones; sabe manejar las emociones negativas de los profesionales; acepta las dificultades de sus hijos; tiene más recursos y apoyos para solucionar los problemas; colabora estrechamente con los profesionales de los diferentes servicios y mantiene una comunicación fluida con ellos; y sabe dar sentido a la enfermedad, salir fortalecida de ésta y crecer como personas (Grau y Fernández, 2IO: 2II).

La resiliencia familiar tiene repercusiones positivas en la familia y en los servicios públicos. En la familia, en cuanto que sus miembros tienen un mejor rendimiento (escolar y laboral), y un mejor bienestar personal y emocional, con la consiguiente mejora de la calidad de vida en su conjunto. Asimismo, se utilizan menos servicios especializados o extraordinarios en el sistema sanitario, educativo y social, lo que constituye un ahorro en el gasto público (Grau y Fernández, 210: 2II). 


\section{Referencias bibliográficas}

Álvarez, F. et al. (2008): “La práctica pediátrica en un medio cultural plural: una experiencia en curso", Arch. Argent. Pediatr., I06: 236-24I.

Björk, W. et al. (2005): "Striving to survive: Families' lived experiences when a child is diagnosed with cancer", J. Pediatr. Oncol.. Nurs., 22: 265-75.

Black, K. y Lobo, M. (2008): “A conceptual review of family resilience factors", Journal of Family Nursing, I4 (I): 33-55.

Borges, Z. y Silva M.H. (2010): "Promoción de la esperanza y resiliencia familiar. Prácticas apreciativas”, Invest. Educ. Enferm., 28 (2): 250-257.

Brody, A.C. y Simmons L.A. (2007): "Family resilience during childhood cancer: The father's perspective", Journal of Pediatric Oncology Nursing, 24 (3): I 52-I65.

Carter, B. y McGoldrick, M. (2005): The Expanded Family Life Cycle: Individual, Family and Social Perspective, Boston: Pearson Education.

Caruso, A., y Mikulic, I.M. (2010): "Evaluación psicológica de la familia con un bebé en la Unidad de Cuidados Intensivos Neonatológicos (UCIN): construcción de un Inventario para Evaluar el Potencial Resiliente Familiar (IPRF)", Anuario de Investigaciones, Facultad de Psicología de la Universidad de Buenos Aires, I7.

Cruz, M (2004): “Un renovado reto en la formación pediátrica: la relación médicopaciente”, Educ. Med., 7: I I I-I 24.

Díaz, C. A. (I999): "Educación sanitaria a padres y niños con asma”, Formación Médica Continuada en Atención Primaria, 6: I9I-202.

Dunst, C.J. et al. (2002): "Family-oriented program models and professional helpgiving practices", Family Relations, 5I (3): 22I-229.

Falicov, C.J. (2007): "Working with transnational immigrants: Expanding meanings of family, community and culture", Fam. Proc., 46 (2): I 57-I 70 .
- (I995): “Training to think culturally: A multidimensional comparative framework", Fam. Proc., 34: 373-388.

Fernández, M. et al. (2OI2): "Impacto de la enfermedad de Hungtinton en la familia", An. Sist. Sanit. Navar., 35 (2): 295-307.

Freixas, M. (200I): "La Educación Especial en el ámbito familiar en Salvador”, en Salvador Mata, F. (coord.), Enciclopedia psicopedagógica de necesidades educativas especiales, Málaga: Aljibe, tomo I: I37-I 55.

Gaite, L. et al. (2008): "Necesidades de los pacientes pediátricos con enfermedades raras durante la edad pediátrica”, An. Sist. Sanit. Navar., 3 I (2): I65-I75.

Gardner, D. et al. (2008): “The development and validation of the inventory of family protective factors: A brief assessment for family counseling", The Family Journal, I6: I07-I I7.

Gómez, E. y Kotliarenco, M.A. (20I0): “Resiliencia familiar: un enfoque de investigación e intervención en familias multiproblemáticas”, Revista de Psicología, I9 (2): I03-I3 I.

Grau, C. (20I2): “Alumnado con tumores intracraneales: papel de la escuela en la mejora de la calidad de vida y en la rehabilitación de los efectos tardíos de la enfermedad y los tratamientos”, Educatio Siglo XXI, 30 (I): I $59-$ I 83 .

- (2004): Atención educativa al alumnado con enfermedades crónicas y de larga duración, Málaga: Aljibe.

Grau, C. y Espada, M.C. (20I2): "Percepciones de los padres de niños enfermos de cáncer sobre los cambios en las relaciones familiares", Psicooncología, 9 (I): I 25-I36.

Grau, C. y Fernández, M. (20I0): "Familia y enfermedad crónica pediátrica”, An. Sist. Sanit. Navar., 33 (2): 203-2I 2.

Grau, C. et al. (2010): "Relaciones padresmédicos en oncología pediátrica: un enfoque cualitativo", An. Sist. Sanit. Navar., 33 (3): 277-285. 
Guralnick, M. J. (2005): “An overview of the developmental systems model for early intervention”, en Guralnick, M.J., The Developmental Systems Approach to Early Intervention, Baltimore: Brooke, 3-28.

Herzer, M. et al. (2010): "Family functioning in the context of pediatric chronic conditions", J. Dev. Behav. Pediatr., $3 \mathrm{I}(\mathrm{I}): 26-34$.

Masten, H.S. et al. (2009): "Resilience in development”, en Lopez, S.J. y Synder, C.R., The Oxford Handbook of Positive Psychology, Oxford: Oxford University Press, I I7-I 3 I.

McCubbin, M. et al. (2002): "Family resilience in childhood cancer", Family Relations, 5 I (2): IO3-III.

McGoldrick, M. et al. (2005): Ethnicity and Family Therapy, New York: The Guilford Press.

Medina-Sanson, A. et al. (2006): "Obstinación terapéutica”, Bol. Med. Hosp. Infant. Mex., 63: 287-290

Muñoz, V. y Sotelo, F.P. (2005): "Educar en la resiliencia. Un cambio de mirada en la prevención de situaciones de riesgo social", Revista Complutense de Educación, I6 (I): IO7-I 24.

Orbuck, T.L. et al. (2005): "Parent-child relationships' and quality of live: Resilience among childhood cancer survivors", Family Relations, 54: I7I-I 83 .

Patterson, J.M. (2002): "Integrating family resilience and family stress theory", Journal of Marriage and Family, 64: 349-360.

Patterson. J.M. et al. (2003): “The impact of childhood cancer on the family: A qualitative analysis of strains, resources, and coping behaviors", Psychooncology, I3: 390-407.

Prado, C. et al. (2007): "La comunicación en oncología pediátrica desde el punto de vista del cirujano", Psicooncología, 4: 197-202.

Presbury, J. et al. (2008): Beyond Brief Counseling and Therapy: An Integrative Approach, Boston: Pearson.

Rolland, J.S. (I994): Families, Illness \& Disabilities: An Integrative Model, New York: Basic Brooks.

Rolland, J.S y Walsh, F. (2006): “Facilitating family resilience with childhood illness and disabilities”, Curr. Opin. Pediatr., I 8: 527-538.

Steinglass, P. (I998): "Multiple family discussion groups for patients with chronic medical illness", Family Systems Health, I6 (I y 2): 55-7I.

Villalba, C (2003): “El concepto de resiliencia individual y familiar. Aplicaciones en la intervención social”, Intervención Psicosocial, I 2 (3): 283-299.

Walsh, F. (2003): "Family resilience: A framework for clinical practice", Fam. Process., 42 (I): I-I 8 .

- (2002): "A family resilience framework: Innovative practice applications", Fam. Relations., 5I (2): I30-I37.

- (I996): "The concept of family resilience: Crisis and challenge", Fam. Process., 35: 26I-28I.

Wong, M. y Chan, S. (2006): “The qualitative experience of Chinese parents with children diagnosed of cancer", J. Clin. Nurs., I 5: 710717 . 